\title{
Combining Ability and Hybrid Vigor for some Root and Vegetative Traits of Rice Under Well Irrigation and Moisture Stress Conditions El-Adl, A.M. M. ${ }^{1}$; A. H. Abd El-Hadi' ${ }^{1}$ A. E. Draz ${ }^{2}$ and Rehab M. H. G. Refai ${ }^{2}$ \\ ${ }^{1}$ Genetics Department, Fac. of Agric., Mansoura Univ., Mansoura, Egypt. \\ ${ }^{2}$ Rice Research Section, Field Crops Research Institute, ARC, Giza, Egypt.
}

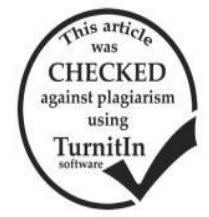

\section{ABSTRACT}

This study was conducted in rice through a $6 \times 6$ diallel set analysis excluding reciprocals involving six rice diverse parents for some root and vegetative traits at the Trial Farm of Rice Research and Training Center, Sakha, Kafr El-Sheikh, Egypt during 2010 and 2011 rice growing seasons. This investigation aimed to assessment the combining ability and heterosis for root and vegetative traits of some rice genotypes at well watering and water stress conditions. The ordinary analysis of variance engaged highly significant differences among genotypes for all studied root and vegetative traits. In addition, both general and specific combining abilities variances were highly significant for all studied traits at both conditions, demonstrating the importance of additive and non-additive genetic variances in determining the performance of these studied traits. Ratios of GCA/SCA were greater than unity for all studied traits, except root volume and number of tillers/plant at normal condition, as well as root/ shoot ratio at drought stress condition, which were less than unity, representative the importance of additive genetic variance in the inheritance of these traits. The greatest general combiners (GCA effects) were IR 7887 for number of tillers/ plant and days to heading (earliness) at both conditions; IR 7887-176-B-2-B and IR 81025B-347-3 under normal irrigation, as well as the AZUCENA and IR 7887-176-B-2-B under drought for root volume. This result indicating that these entries could be considered as good general combiners for the improvement of these root and vegetative traits. The greatest cross combinations (SCA effects) were: Sakha101 X IR 81025-B-347-3 at normal irrigation and Giza177 X IR7887-176-B-2-B at drought stress for number of tillers/ plant; IR 7887-176-B-2-B X IR 80508-B-194-1-B under normal irrigation and Giza 177 X IR 7887-176-B-2-B under drought stress for days to heading (earliness); Sakha101 X AZUCENA and Sakha 101 X IR7887-176-B-2-B under both conditions for root volume; Sakha101 X IR 80508-B-194-1-B and Sakha101 X Azucena under both conditions for maximum root length; Giza 177 X Sakha101, IR7887-176-B-2-B X IR81025-B-347-3 and IR80508-B-194-B X IR81025-B-k347-3 under both conditions for plant height (shortness. This recommended that these crosses are useful for improvement these traits. The highest significant heterobeltiosis $\left(\mathrm{H}_{\mathrm{B} . \mathrm{P}} \%\right)$ in the desirable direction under drought condition were recorded by: Sakha101 X IR 80508-B-194-1B for root volume; Sakha101 X IR 80508-B-194-1-B for maximum root length; Sakha101 X IR 81025-B-347 for number of tillers /plant. Higher heritability assessments in broad sense $\left(\mathrm{h}_{\text {B.S }} \%\right)$ were detected for root and vegetative traits, demonstrating that genetic variance played the main part of phenotypic variance.

Keywords: rice, drought, combining ability, hybrid vigor and heritability

\section{INTRODUCTION}

Rice (Oryza sativa, L.) is one of the most important staple grains consumed by about half of the world's population, providing sufficient energy in the form of calories and a good source of thiamine, riboflavin and niacin (Zhu et al., 2010). Rice cultivation is the main activity and source of income for millions of households around the world and many countries in Asia and Africa, which rely heavily on rice as a source of foreign exchange earnings and government revenues. In addition, rice is one of the main field crops in Egypt. Although rice cultivated area is about 1.600 million faddan, which produced about 6.300 million tons of rice rice with an average of 3.938 tons / faddan in 2016 season (FAO, 2018).

Drought is one of the major abiotic stresses that limit rice production in the world. About $30 \%$ of the world's rice-producing regions suffer from moisture stress and water shortages, at both in rainfed and irrigated areas. About 18 million tons of rice valued at US 650 million American dollar is lost annually due to drought, (Pandey et al., 2005). For this reason, breeding for drought tolerance has become a top priority in the rice-breeding program, especially under Egyptian conditions. The total water needs of the rice crop are a serious problem due to the limited irrigation of water available in the Nile River. Some rice-growing areas, especially those at the end of final irrigation water, fall through different growth stages, which is one of the most serious constraints to rice production (Abdullah et al., 2009).

Rich and better-quality rice varieties cannot be developed without prior knowledge of their genetic characteristics. Therefore, with the help of appropriate quantitative genetic methods, breeders try to combine the required characteristics of different species. Therefore, the current research aimed to assessment the general and specific combining abilities, nature of gene action, heterosis over better parent (heterobeltiosis) for root and vegetative traits of rice genotypes cultivated under well irrigation and water stress environments.

\section{MATERIALS AND METHODS}

The present study was conducted at the Trial Farm of the Rice Research and Training Center (RRTC), Sakha, Kafrelsheikh, Field Crops Research Institute, (FCRI), Agricultural Research Center (ARC) Giza, Egypt, during two successive rice-growing seasons of 2010 and 2011. The target of this study was to define the inheritance of root and vegetative traits, which associated with drought tolerance. Six parents are used namely; Giza177, Sakha101, Asucena, IR78875-176-B-2-B, IR81025-B-2-7$3 \mathrm{a}$ and IR80508-B-194-1-B. These genotypes differed in their reaction to drought stress.

The six rice parents were grown in three dates in 2010 season with 10 days intervals to overawe the difference of heading date among them. Thirty days after sowing, seedlings of each parent was individually transplanted in the experimental field in three rows (each row five maters long) with plant spacing $20 \times 20 \mathrm{~cm}$. A half diallel cross was carried out among the six parents at flowering to produce $15 \mathrm{~F}_{1}$ hybrids seeds (without reciprocals ). Bulk emasculation method was practiced by using hot water technique according to Jodon (1938) and modified by Butany (1961). The hybrid seeds were grown in 2011 rice growing season as $F_{1}$ plants in May $7^{\text {th }}$ and 
plants were transplanted individually after 30 days from sowing in a Randomized Complete Block Design (RCBD), with three replications, each replicate consisted of one row for each parent, and three rows for each $F_{1}$ plants hybrid. Each row was five meters long and contained 25 individual plants. All genotypes (six parents and their $15 \mathrm{~F}_{1}$ crosses) were planted in two Experiments; first one both normal irrigation (irrigation every four days) and second one drought conditions (drought stress was imposed by using flush irrigation every 12 days without standing water after irrigation). All recommended agriculture practices were accomplished as usual for the ordinary rice field. The data were verified on an individual plants basis for parents and their $15 \mathrm{~F}_{1}$ crosses. Studied traits were: root volume $\left(\mathrm{cm}^{3}\right)$, root/ shoot ratio, root length $(\mathrm{cm})$, number of tillers/plant, days to heading (days), plant height $(\mathrm{cm})$.

The combining ability analysis was completed following Griffing's (1956), Model 1, Method 2, where parents, and one set of $F_{1}$ 's were included to assessment the effects of general (GCA) and specific (SCA) combining abilities and variance components under normal and water stress conditions. The GCA/ SCA ratio was assessed to measure the relative importance of additive gene or non-additive gene effects (Singh and Chaudhary, 1979). Hybrid vigor was assessed according to Falconer and Mackay (1996). Furthermore, appropriate LSD standards were premeditated to test the significance of heterotic effects according to the formula suggested by Wynne et al., (1970).

\section{RESULTS AND DISCUSSION}

\section{Analysis of Variance:}

Analysis of variance signposted highly significant differences among genotypes for all root and morphological traits (Table, 1) under both well irrigation and drought stress conditions. The results of combining ability analysis of genotypes indicated could be used in hybrid breeding program to facilitate the breeder's decision when they are selecting the cultivars. Combining ability analysis of cultivars is necessary to exploit the relevant type of gene action for a breeding program.

Both GCA and SCA variances were found to be highly significant for all root and morphological traits at both well watering and drought situations. This would indicate the importance of additive and non-additive genetic variances in determining the performance of these studied traits (Table, 1). These findings are similar with those reported by Abd El-Lattef et al., (2012), Sultan et al., (2014 a, b \& c), Ghosh et al., (2014), Karpagam et al., (2016), Abdel-Moneam et al., (2016), Hefena et al., (2016), Malemba et al., (2017) and Ramesh et al., (2018), who revealed that both GCA and SCA were significant, demonstrating the importance of both additive and nonadditive gene action in the expression of studied vegetative, physiological and yield traits of their studied rice genotypes.

GCA/SCA ratio was used to explain the nature of gene action involved. The most of GCA/SCA ratios were found to be greater than unity for all studied traits (Table, 1), except number of tillers/plant and root volume at normal condition, and root shoot ratio at drought stress condition, were found to be less than unity, representing the prominence of non-additive genetic variance in the inheritance of these traits, indicates that additive and additive $\mathrm{x}$ additive types of gene actions were superior importance in the inheritance of studied traits, so the selection based on the accumulation of additive effect, would be successful in improving these vegetative and yield attributed behaviors. Significant general and specific combining ability variances for such characters were reported by Abd El-Lattef et al., (2012), Karpagam et al., (2016), Sultan et al., (2014 a, b \& c), Abdel-Moneam et al., (2016), Hefena et al. (2016), Ghosh et al., (2014), Malemba et al., (2017) and Ramesh et al., (2018), who revealed that most of GCA/SCA ratios were found to be greater than unity for most studied vegetative, physiological traits of their studied rice genotypes.

Table 1. Analysis of variance for ordinary analysis and combining ability for root and vegetative traits of rice genotypes under well irrigation and drought environments.

\begin{tabular}{|c|c|c|c|c|c|c|c|}
\hline \multirow{2}{*}{$\begin{array}{l}\text { Traits } \\
\text { S.O.V }\end{array}$} & \multirow{2}{*}{ d.f } & \multicolumn{2}{|c|}{ Max. root length $(\mathrm{cm})$} & \multicolumn{2}{|c|}{ Root volume $\left(\mathrm{cm}^{3}\right)$} & \multicolumn{2}{|c|}{ Root / shoot ratio } \\
\hline & & Normal & Drought & Normal & Drought & Normal & Drought \\
\hline Rep. & 2 & 1.76 & 4.30 & 0.00 & 0.01 & 0.043 & $0.001 * *$ \\
\hline Geno. & 20 & $68.78 * *$ & $28.68 * *$ & $99.50 * *$ & $31.33 * *$ & $0.08 * *$ & $0.01 * *$ \\
\hline Error & 40 & 4.08 & 4.60 & 0.0001 & 0.0001 & 0.02 & 0.01 \\
\hline GCA & 5 & $35.76 * *$ & $14.13 * *$ & $29.93 * *$ & $19.04 * *$ & $0.04 * *$ & $0.04 * *$ \\
\hline SCA & 15 & $18.65 * *$ & $8.04 * *$ & $34.24 * *$ & $7.58 * *$ & $0.02 * *$ & $0.02 * *$ \\
\hline Error & 40 & 1.36 & 1.53 & 0.00001 & 0.00001 & 0.01 & 0.00001 \\
\hline GCA/SCA & - & 1.92 & 1.76 & 0.87 & 2.51 & 1.49 & 0.99 \\
\hline \multirow{2}{*}{ S.O.V } & \multirow{2}{*}{ d.f } & \multicolumn{2}{|c|}{ No. of tillers/plant } & \multicolumn{2}{|c|}{ Days to heading (day) } & \multicolumn{2}{|c|}{ Plant height $(\mathrm{cm})$} \\
\hline & & Normal & Drought & Normal & Drought & Normal & Drought \\
\hline Rep. & 2 & 2.093 & 0.70 & 66.05 & 14.28 & $53.41 * *$ & $1.71 * *$ \\
\hline Geno. & 20 & $43.71 * *$ & $10.28 * *$ & $258.68 * *$ & $217.77 * *$ & $1281.6 * *$ & $1161.9 * *$ \\
\hline Error & 40 & 4.24 & 3.91 & 52.95 & 13.98 & 5.93 & 0.214 \\
\hline GCA & 5 & $9.34 * *$ & $4.03 *$ & $244.36 * *$ & $176.81 * *$ & $1337.60 * *$ & $1130.45^{* *}$ \\
\hline SCA & 15 & $16.31 * *$ & $3.23 *$ & 33.52 & $37.85 * *$ & $123.75 * *$ & $139.59 * *$ \\
\hline Error & 40 & 1.41 & 1.30 & 17.65 & 4.66 & 1.97 & 0.07 \\
\hline GCA/SCA & - & 0.57 & 1.25 & 7.29 & 4.67 & 10.81 & 8.10 \\
\hline
\end{tabular}

*, ** Significant at 0.05 and 0.01 levels of probability, respectively. 


\section{Effects of general combining ability (GCA):}

General combining ability effects calculated herein were found to differ significantly from zero in most cases. Highly significant and positive values (GCA) would be in some characters, such as the characters that have high involvement to yield, however, high negative values for days to heading and plant height would be useful from the breeder's point view of general combining ability effects (gi) of each parent for all characters, which are useful for drought tolerance contributing variation of drought tolerance at both environs are presented in Table 2. Such effects are being used to compare the average presentation of each parent with other parents and facilitate selection of parents for further improvement to drought resistance.

The greatest general combiners were parental variety Giza177 for number of tillers/ plant under both well watering and water stress; the parents Giza 177, IR 80508B-194-1-B and IR 81025-B-347-3 for days to heading (earliness) at both environments. The two varieties IR 7887-176-B-2-B and IR 81025-B-347-3 under well irrigation and the parents AZUCENA and IR 7887-176-B2-B under water stress were the best combiners for root volume; while the variety Azucena under both situations and IR 7887-176-B-2-B under drought for root length. In addition, the best general combiners were Giza177 and Sakha101 for plant height (shortness) at the both conditions; Azucena at well irrigation and IR81025-B-3473 at drought stress for root/shoot ratio. This result demonstrating that these entries could be reflected as good general combiners for the improvement of these root and vegetative characters. These results were in conformity with those reported previously by Yogameenashi et al., (2003), Manickavelu et al., (2006) and Abd El-Lattef and Mady (2009) under both conditions. They reported that additive gene effects played an important role in the inheritance of these behaviors.

Table 2. Estimates of G.C.A. effects of the six parents' rice genotypes for root and vegetative traits under well irrigation and drought environments.

\begin{tabular}{|c|c|c|c|c|c|c|}
\hline \multirow{2}{*}{ Parents } & \multicolumn{2}{|c|}{ Max. root length $(\mathrm{cm})$} & \multicolumn{2}{|c|}{ Root volume $\left(\mathrm{cm}^{3}\right)$} & \multicolumn{2}{|c|}{ Shoot / root ratio } \\
\hline & Normal & Drought & Normal & Drought & Normal & Drought \\
\hline Giza 177 & $-3.83 * *$ & $-1.61 *$ & $-3.05 * *$ & $-1.09 * *$ & $-0.06 *$ & $-0.02 * *$ \\
\hline Sakha101 & -0.31 & $-1.39 *$ & $-0.83 * *$ & $-1.16^{* *}$ & -0.03 & 0.01 \\
\hline AZUCENA & $2.60 * *$ & $1.75^{* *}$ & $-0.12 * *$ & $2.63 * *$ & $0.13 * *$ & 0.01 \\
\hline IR 7887-176-B-2-B & 0.70 & $1.08 * *$ & $2.52 * *$ & $1.13 * *$ & -0.02 & 0.00 \\
\hline IR 80508-B-194-1-B & 0.40 & -0.07 & $-0.05 * *$ & $-0.56 * *$ & -0.03 & $-0.02 * *$ \\
\hline IR 81025-B-347-3 & 0.44 & 0.24 & $1.53 * *$ & $-0.94 * *$ & 0.02 & $0.02 * *$ \\
\hline $\mathrm{LSD}_{0.05}$ & 0.76 & 0.81 & 0.02 & 0.02 & 0.06 & 0.01 \\
\hline $\mathrm{LSD}_{0.01}$ & 1.02 & 1.08 & 0.03 & 0.03 & 0.07 & 0.02 \\
\hline
\end{tabular}

*, ** Significant at 0.05 and 0.01 levels of probability, respectively.

Table 2. Continued.

\begin{tabular}{|c|c|c|c|c|c|c|}
\hline \multirow{2}{*}{ Parents } & \multicolumn{2}{|c|}{ Number of tillers/plant } & \multicolumn{2}{|c|}{ Days to heading (day) } & \multicolumn{2}{|c|}{ Plant height $(\mathrm{cm})$} \\
\hline & Normal & Drought & Normal & Drought & Normal & Drought \\
\hline Giza 177 & $0.82 *$ & $0.87 *$ & $-5.08 * *$ & $-5.13 * *$ & $-17.05 * *$ & $-16.48 * *$ \\
\hline Sakha101 & 0.76 & -0.26 & $8.67 * *$ & $5.58 * *$ & $-15.42 * *$ & $-13.83 * *$ \\
\hline AZUCENA & $-1.77 * *$ & $-0.98 *$ & 0.17 & $2.54 * *$ & $13.75 * *$ & $10.74 * *$ \\
\hline IR 7887-176-B-2-B & 0.36 & 0.46 & $4.25 * *$ & $3.92 * *$ & $6.59 * *$ & $6.89 * *$ \\
\hline IR 80508-B-194-1-B & $-0.90 *$ & -0.56 & $-3.08 *$ & $-1.54 *$ & $5.21 * *$ & $5.86 * *$ \\
\hline IR 81025-B-347-3 & 0.73 & 0.46 & $-4.92 * *$ & $-5.38 * *$ & $6.91 * *$ & $6.82 * *$ \\
\hline $\mathrm{LSD}_{0.05}$ & 0.78 & 0.75 & 2.74 & 1.41 & 0.92 & 0.17 \\
\hline $\mathrm{LSD}_{0.01}$ & 1.04 & 0.99 & 3.67 & 1.89 & 1.23 & 0.23 \\
\hline
\end{tabular}

*, ** Significant at 0.05 and 0.01 levels of probability, respectively.

\section{Effects of Specific combining ability:}

Estimations of specific combining ability (SCA) effects for the hybrid combinations at the two well watering and drought stress environments are given in Table 3. The best cross mixtures for improving root and vegetative characters were: the crosses Sakha101 X IR 81025-B-347-3 at normal irrigation and Giza177 $\mathrm{X}$ IR7887-176-B-2-B at drought stress for number of tillers/plant; IR 7887-176-B-2-B X IR 80508-B-194-1-B under well watering and Giza 177 X IR 7887-176-B-2-B under drought stress for heading date (earliness); Sakha101 X Azucena and Sakha 101 X IR7887-176-B-2-B under both situations for root volume; Sakha101 X IR 80508-B194-1-B and Sakha101 X Azucena under both environments for maximum length of root. In addition, the best cross combinations were Giza 177 X Sakha101, IR7887-176-B-2-B X IR81025-B-347-3 and IR80508-B194-B X IR81025-B-k347-3 under both situations for height of plant (shortness). As well as, five crosses: Giza 177 X Azucena, Sakha101 X Azucena, Sakha101 X IR 7887-176-B-2-B, Sakha101 X IR 80508-B-194-1-B and IR 7887-176-B-2-B X IR 81025-B-347-3 were the best combinations under drought for ratio of root/shoot. Significant SCA effects in positive direction for these traits in some studied rice cross combinations under both conditions were reported by many researchers, such as Manickavelu et al., (2006) , Abd-Allah et al., (2009), Abd El-Lattef et al., (2012), Sultan et al., (2014 a, b \& c), Malemba et al., (2017) and Ramesh et al., (2018). 
Table 3. Estimates of S.C.A. effects of $15 \mathrm{~F}_{1}$ rice crosses for root and vegetative traits under well irrigation and drought environments.

\begin{tabular}{lcccccc}
\hline Traits & \multicolumn{2}{c}{ Maximum root length $(\mathbf{c m})$} & \multicolumn{2}{c}{ Root volume $\left(\mathbf{c m}^{3}\right)$} & \multicolumn{2}{c}{ Shoot/root ratio } \\
\hline Crosses & Normal & Drought & Normal & Drought & Normal & Drought \\
\hline Giza177 X Sakha101 & $-3.00^{* *}$ & -0.69 & $-8.07^{* *}$ & $-0.50^{*}$ & -0.04 & $-0.02^{*}$ \\
Giza177 X AZUCENA & $-3.14^{* *}$ & 0.39 & $1.66^{* *}$ & -0.01 & -0.10 & $0.05^{* *}$ \\
Giza177 X IR 7887-176-B-2-B & $4.48^{* *}$ & 2.17 & $7.03^{* *}$ & $1.05^{*}$ & 0.07 & $-0.04^{* *}$ \\
Giza177 X IR 80508-B-194-1-B & 1.28 & 0.66 & $5.60^{* *}$ & $0.07^{*}$ & -0.05 & -0.02 \\
Giza177 X IR 81025-B-347-3 & 1.24 & 1.29 & $0.35^{* *}$ & $1.40^{* *}$ & -0.03 & $-0.04^{* *}$ \\
Sakha101 X AZUCENA & $7.58^{* *}$ & $5.61^{* *}$ & $8.22^{* *}$ & $3.95^{* *}$ & $-0.15^{*}$ & $0.03^{* *}$ \\
Sakha101 X IR 7887-176-B-2-B & $2.91^{* *}$ & -0.28 & $10.80^{* *}$ & $3.12^{* *}$ & 0.13 & $0.08^{* *}$ \\
Sakha101 X IR 80508-B-194-1-B & $7.65^{* *}$ & $3.21^{* *}$ & $0.71^{* *}$ & $2.91^{* *}$ & 0.11 & $0.06^{* *}$ \\
Sakha101 X IR 81025-B-347-3 & $-2.40^{*}$ & 0.23 & $-1.09^{* *}$ & $0.62^{* *}$ & 0.06 & $-0.04^{* *}$ \\
AZUCENA X IR7887-176-B-2-B & -0.16 & 2.00 & $0.65^{* *}$ & $0.53^{* *}$ & -0.20 & 0.02 \\
AZUCENA X IR80508-B-194-1-B & $3.31^{* *}$ & 0.07 & $-3.78^{* *}$ & $-0.87^{* *}$ & $-0.17^{*}$ & -0.01 \\
AZUCENA X IR81025-B-347-3 & -1.02 & $-5.46^{* *}$ & $3.53^{* *}$ & $-5.71^{* *}$ & $-0.21^{* *}$ & -0.02 \\
IR7887-176-B-2-B X IR80508-B-194-1-B & $-4.80^{* *}$ & -1.15 & $-6.19^{* *}$ & $-1.26^{* *}$ & -0.01 & 0.01 \\
IR7887-176-B-2-B X IR81025-B-347-3 & 1.85 & -1.80 & $-3.44^{* *}$ & $-1.21^{* *}$ & 0.05 & $0.07^{* *}$ \\
IR80508-B-194-1-B X IR81025-B-347-3 & -2.05 & 1.42 & $3.79^{* *}$ & $2.52^{* *}$ & 0.0 & $-0.03^{* *}$ \\
\hline LSD $_{0.05}$ & 2.090 & 2.219 & 0.057 & 0.057 & 0.150 & 0.031 \\
LSD $_{0.01}$ & 2.796 & 2.968 & 0.076 & 0.076 & 0.201 & 0.042 \\
\hline
\end{tabular}

Table 3. Continued.

\begin{tabular}{lcccccc}
\hline Traits & \multicolumn{2}{c}{ Number of tillers/plant } & \multicolumn{2}{c}{ Days to heading (day) } & \multicolumn{2}{c}{ Plant height (cm) } \\
\cline { 2 - 7 } Crosses & Normal & Drought & Normal & Drought & Normal & Drought \\
\hline Giza177 X Sakha101 & $2.63^{*}$ & -0.68 & -0.11 & 2.78 & $-11.41^{* *}$ & $-8.61^{* *}$ \\
Giza177 X AZUCENA & 0.16 & 0.18 & -6.61 & -3.51 & -0.99 & $6.42^{* *}$ \\
Giza177 X IR 7887-176-B-2-B & $6.37^{* *}$ & $2.74^{* *}$ & -5.69 & $-8.55^{* *}$ & $3.77^{* *}$ & $7.87^{* *}$ \\
Giza177 X IR 80508-B-194-1-B & $2.29^{*}$ & 0.36 & 1.98 & -0.43 & $15.55^{* *}$ & $-0.70^{* *}$ \\
Giza177 X IR 81025-B-347-3 & -1.54 & 1.81 & -2.19 & 1.40 & $5.85^{* *}$ & 0.14 \\
Sakha101 X AZUCENA & $-5.38^{* *}$ & 0.71 & $7.31^{*}$ & 2.78 & $6.39^{* *}$ & $9.77^{* *}$ \\
Sakha101 X IR 7887-176-B-2-B & -0.11 & 1.60 & $8.23^{*}$ & $7.40^{* *}$ & $5.95^{* *}$ & $5.02^{* *}$ \\
Sakha101 X IR 80508-B-194-1-B & 1.22 & 0.28 & -1.77 & $11.86^{* *}$ & $17.93^{* *}$ & $15.65^{* *}$ \\
Sakha101 X IR 81025-B-347-3 & $7.58^{* *}$ & $2.66^{*}$ & -1.27 & $-4.30^{*}$ & $11.23^{* *}$ & $4.69^{* *}$ \\
AZUCENA X IR 7887-176-B-2-B & 1.15 & -1.41 & -0.27 & 1.45 & $2.70^{*}$ & $7.05^{* *}$ \\
AZUCENA X IR 80508-B-194-1-B & $-2.52^{*}$ & 0.54 & -5.61 & $-5.10^{*}$ & $8.55^{* *}$ & $20.01^{* *}$ \\
AZUCENA X IR 81025-B-347-3 & $2.84^{* *}$ & 1.92 & -1.11 & -3.26 & $5.05^{* *}$ & $5.12^{* *}$ \\
IR 7887-176-B-2-B X IR 80508-B-194-1-B & -1.25 & 0.30 & $-9.02^{*}$ & $-5.80^{* *}$ & -0.02 & $-2.68^{* *}$ \\
IR 7887-176-B-2-B X IR 81025-B-347-3 & -1.15 & -0.99 & 1.48 & 3.36 & $-5.19^{* *}$ & $-5.43^{* *}$ \\
IR 80508-B-194-1-B X IR 81025-B-347-3 & $-5.49^{* *}$ & -0.10 & -2.19 & -3.85 & $-7.00^{* *}$ & $-4.60^{* *}$ \\
\hline LSD 0.05 & 2.129 & 2.046 & 7.526 & 3.868 & 2.517 & 0.479 \\
LSD 0.01 & 2.849 & 2.737 & 10.069 & 5.175 & 3.368 & 0.641 \\
\hline
\end{tabular}

*, ** Significant at 0.05 and 0.01 levels of probability, respectively.

Heterosis over the best parent (Heterobeltiosis, $\mathbf{H}_{\text {B.P }}$ $\%)$ :

Estimations of heterotic effects of $15 \mathrm{~F}_{1}$ single crosses of rice over superior parent $\left(\mathrm{H}_{\mathrm{B} . \mathrm{P}} \%\right)$ for all root and morphological characters under well irrigation and water stress environments are presented in Table 4.

The highest significant heterosis estimates over better parent (heterobeltiosis, $\mathrm{H}_{\text {B.P }} \%$ ) in the desirable direction were recorded by: Giza 177 X IR 7887-176-B-2B $(63.04 \%)$ under well irrigation, Sakha101 X IR 81025 B-347-(363.89\%) under drought stress for number of tillers /plant. Only one cross Azucena X IR 80508-B-1941-B $(-10.56 \%)$ under normal condition showed heterobeltiosis for days to heading (earliness). While, Sakha101 X AZUCENA (47.67\%) under normal irrigation and Sakha101 X IR 80508-B-194-1-B (21.18 \%) under drought stress condition for root volume. However,
Sakha101 X IR 80508-B-194-1-B (37.52 \%) under normal irrigation and Sakha101 X IR 80508-B-194-1-B (21.18 \%) under drought showed heterobeltiosis for maximum root length. On the other hand, no hybrids showed desirable negative significant heterosis $\left(\mathrm{H}_{\mathrm{B} . \mathrm{P}} \%\right)$ under both environments for height of plant (shortness); and also there were not any crosses showed desirable positive significant heterosis $\left(\mathrm{H}_{\text {B.P }} \%\right)$ effects under well irrigation or drought environments for ratio of root/shoot.

These findings exposed that over dominance frolicked a significant part in the inheritance of these characters in these crosses. Parallel results were attained beforehand by Gaballah (2009), Abd Allah (2010), Yadav et al., (2010), Tiwari et al., (2011) and El-Gamal (2013), who found positive significant heterosis over better parent for some crosses for the same characters under both well irrigation and drought conditions. 
Table 4. Heterotic effects of $15 F_{1}$ single crosses of rice over better parent $\left(H_{B . P} \%\right)$ for root and vegetative traits under well irrigation and drought environments.

\begin{tabular}{|c|c|c|c|c|c|c|}
\hline \multirow{2}{*}{$\begin{array}{l}\text { Trait } \\
\text { Cross }\end{array}$} & \multicolumn{2}{|c|}{ Max. root length $(\mathrm{cm})$} & \multicolumn{2}{|c|}{ Root volume $\left(\mathrm{cm}^{3}\right)$} & \multicolumn{2}{|c|}{ Root : shoot ratio } \\
\hline & Normal & Drought & Normal & Drought & Normal & Drought \\
\hline Giza177 X Sakha101 & -0.73 & 9.06 & $-20.00 * *$ & $2.38 * *$ & -32.00 & -0.01 \\
\hline Giza177 X AZUCENA & $-21.29 * *$ & -7.12 & $14.61 * *$ & $-17.07 * *$ & $-72.45 * *$ & 0.05 \\
\hline Giza177 X IR 7887-176-B-2-B & 7.79 & -0.22 & $18.06 * *$ & $-0.22 * *$ & 16.00 & -0.04 \\
\hline Giza177 X IR 80508-B-194-1-B & -0.97 & 6.46 & $8.37 * *$ & $6.46^{* *}$ & -40.74 & -0.05 \\
\hline Giza177 X IR 81025-B-347-3 & $-14.26 * *$ & -11.48 & $-7.95 * *$ & $0.29 * *$ & -39.47 & -0.12 \\
\hline Sakha101 X AZUCENA & $27.02 * *$ & $16.20 *$ & $47.67 * *$ & $-3.18 * *$ & $-74.49 * *$ & 0.08 \\
\hline Sakha101 X IR 7887-176-B-2-B & $15.09 * *$ & -9.94 & $36.49 * *$ & $8.54 * *$ & 62.50 & 0.12 \\
\hline Sakha101 X IR 80508-B-194-1-B & $37.52 * *$ & 21.18 & 0.00 & $21.18 * *$ & 29.63 & 0.09 \\
\hline Sakha101 X IR 81025-B-347-3 & $-14.63 * *$ & $-14.97 *$ & $-5.62 * *$ & $-3.71 * *$ & -7.89 & -0.08 \\
\hline AZUCENA X IR 7887-176-B-2-B & $4.14 * *$ & 11.36 & $7.49 * *$ & $-7.21 * *$ & $-77.55 * *$ & 0.05 \\
\hline AZUCENA X IR 80508-B-194-1-B & 14.92 & -1.89 & $-11.89 * *$ & $-18.25 * *$ & $-76.53 * *$ & 0.01 \\
\hline AZUCENA X IR 81025-B-347-3 & $-0.20 * *$ & $-25.69 * *$ & $10.32 * *$ & $-36.89 * *$ & $-75.51 * *$ & -0.07 \\
\hline IR 7887-176-B-2-B X IR 80508-B-194-1-B & -10.99 & -8.02 & $-13.33 * *$ & $-8.02 * *$ & -11.11 & 0.04 \\
\hline IR 7887-176-B-2-B X IR 81025-B-347-3 & 3.07 & $-13.08 *$ & $-2.63 * *$ & $-9.46 * *$ & -7.89 & 0.02 \\
\hline IR 80508-B-194-1-B X IR 81025-B-347-3 & $-11.05^{*}$ & -4.46 & $11.30 * *$ & $8.19 * *$ & -7.89 & -0.09 \\
\hline $\operatorname{LSD}_{0.05}$ & 2.78 & 2.95 & 0.001 & 0.001 & 0.19 & 0.14 \\
\hline $\mathrm{LSD}_{0.01}$ & 4.00 & 4.24 & 0.002 & 0.002 & 0.28 & 0.20 \\
\hline
\end{tabular}

*, ** Significant at 0.05 and 0.01 levels of probability, respectively.

Table 4. Continued

\begin{tabular}{|c|c|c|c|c|c|c|}
\hline \multirow{2}{*}{$\begin{array}{l}\text { Treats and Treatments } \\
\text { Crosses }\end{array}$} & \multicolumn{2}{|c|}{ No. of tillers/plant } & \multicolumn{2}{|c|}{ Days to heading (day) } & \multicolumn{2}{|c|}{ Plant height (cm) } \\
\hline & Normal & Drought & Normal & Drought & Normal & Drought \\
\hline Giza177 X Sakha101 & $37.48 * *$ & 4.72 & 7.72 & $10.03 * *$ & 2.31 & $2.42 * *$ \\
\hline Giza177 X AZUCENA & 5.83 & 6.26 & -8.07 & 0.00 & $39.35^{* *}$ & $41.14 * *$ \\
\hline Giza177 X IR 7887-176-B-2-B & $63.04 * *$ & $48.91 * *$ & -2.81 & -3.95 & $36.74 * *$ & $38.41 * *$ \\
\hline Giza177 X IR 80508-B-194-1-B & 6.40 & 13.34 & -2.45 & -1.08 & $48.04 * *$ & $27.50 * *$ \\
\hline Giza177 X IR 81025-B-347-3 & -1.95 & $42.50 *$ & -5.45 & -1.82 & $39.35 * *$ & $29.55 * *$ \\
\hline Sakha101 X AZUCENA & $-32.58 * *$ & 37.03 & $12.38 *$ & $10.00 * *$ & $58.43 * *$ & $57.63 * *$ \\
\hline Sakha101 X IR 7887-176-B-2-B & 16.33 & 22.91 & 9.09 & $16.00 * *$ & $49.65 * *$ & $47.22 * *$ \\
\hline Sakha101 X IR 80508-B-194-1-B & $0.00 *$ & 17.81 & 1.65 & $17.74 * *$ & $61.89 * *$ & $58.84 * *$ \\
\hline Sakha101 X IR 81025-B-347-3 & 51.62 & $63.89 * *$ & 10.54 & 3.63 & $56.12 * *$ & 46.73 \\
\hline AZUCENA X IR 7887-176-B-2-B & $9.38 *$ & -19.82 & 0.65 & -0.93 & $9.47 * *$ & $22.68 * *$ \\
\hline AZUCENA X IR 80508-B-194-1-B & -35.48 & 11.22 & $-10.56^{*}$ & -2.73 & $27.59 * *$ & $32.56 * *$ \\
\hline AZUCENA X IR 81025-B-347-3 & $8.68 * *$ & $43.47 *$ & 1.45 & 1.45 & $11.94 * *$ & $21.03 * *$ \\
\hline IR 7887-176-B-2-B X IR 80508-B-194-1-B & -16.24 & 4.58 & -9.90 & -2.05 & $15.05 * *$ & $10.05 * *$ \\
\hline IR 7887-176-B-2-B X IR 81025-B-347-3 & -2.36 & 1.60 & 8.73 & $10.18 * *$ & -0.38 & 0.30 \\
\hline IR 80508-B-194-1-B X IR 81025-B-347-3 & -38.14 & 21.25 & -3.27 & -3.64 & $9.73 * *$ & $8.40 * *$ \\
\hline $\mathrm{LSD}_{0.05}$ & 2.83 & 2.72 & 10.01 & 5.14 & 3.35 & 0.64 \\
\hline $\mathrm{LSD}_{0.01}$ & 4.07 & 3.91 & 14.40 & 7.40 & 4.82 & 0.92 \\
\hline
\end{tabular}

*,** Significant at 0.05 and 0.01 levels of probability, respectively.

\section{Estimates of heritability:}

Estimates of heritability in broad $\left(\mathrm{h}_{\mathrm{B} . \mathrm{S}} \%\right)$ and narrow $\left(\mathrm{h}_{\mathrm{N} . \mathrm{S}} \%\right)$ senses for root and morphological characters under well irrigation and drought are presented in Table 5.

Higher heritability estimates in broad sense $\left(\mathrm{h}_{\mathrm{B} . \mathrm{S}} \%\right)$ were noticed for tillers/plant (91.84 and 63.46\%), heading date (71.47 and $92.15 \%$ ), root volume (99.99 and $99.99 \%$ ), length of root (94.07 and $84.05 \%)$, plant height (99.32 and $99.98 \%)$, root/shoot ratio (75.47 and $89.27 \%$ ) at both well watering and drought, respectively. These results designate that genotypic variances played the major role of phenotypic variances.

Moderate heritability estimates in narrow sense $\left(\mathrm{h}_{\mathrm{N} . \mathrm{S}} \%\right)$ were identified for heading date (45.81 and $36.25 \%)$ and height of plant $(57.43$ and $50.30 \%)$ under well and drought, respectively. These results signposted that selection for these both characters in early segregating generations would be effective in the studied crosses. While, the other studied vegetative traits (tillers/plant, volume of roots, maximum length of roots and ratio of shoot/root) documented lower percentages of narrow sense heritability under both well irrigation and drought, demonstrating that selection for these traits in late segregating generations would be effective in the studied crosses. These results were approved with those achieved by Sedeek (2006), Abd El-Lattef et al. (2012), and Hassan et al., (2016).

Table 5. Heritability estimates in broad $\left(h_{\text {B.S }} \%\right)$ and narrow $\left(h_{\mathrm{N} . \mathrm{S}} \%\right)$ senses for root and vegetative traits under well irrigation and drought environments.

\begin{tabular}{lcccc}
\hline \multirow{2}{*}{ Treatments } & \multicolumn{2}{c}{$\mathbf{h}_{\mathrm{B.S}}$ \% } & \multicolumn{2}{c}{$\mathbf{h}_{\mathrm{N.S}}$ \% } \\
\cline { 2 - 5 } & Normal & Drought & Normal & Drought \\
\hline Maximum root length (cm) & 94.07 & 84.05 & 18.73 & 16.37 \\
Root volume $\left(\mathrm{cm}^{3}\right)$ & 99.99 & 99.99 & 9.85 & 23.89 \\
Root : shoot ratio & 75.47 & 89.27 & 13.11 & 9.77 \\
Number of tillers/plant & 91.84 & 63.46 & 5.73 & 9.54 \\
Days to heading (day) & 71.47 & 92.15 & 45.81 & 36.25 \\
Plant height (cm) & 99.32 & 99.98 & 57.43 & 50.30 \\
\hline
\end{tabular}




\section{REFERENCES}

Abd Allah, A.A. (2010). Development of some high yielding rice lines tolerant to drought stress conditions. J. Med. Plants Res., 4(7): 528-535.

Abd Allah, A.A. ; A.H. Selim and M.A. Abo-Yousef (2009). Genetic variability and inheritance of drought avoidance mechanism in rice (Oryza sativa L.) J. Agric. Res. Kafrelsheikh Univ., 35(2):152162.

Abd El-Lattef, A.S. ; A.A.B. Abo Khalifa and A.A.A. ElGohary (2012). Inheritance of some quantitative characters under drought conditions in rice (Oryza sativa L.). Inter. J. Biology, Pharmacy and Appl. Sci., 1(5): 620- 635.

Abd El-Lattef, A.S. and A.A. Mady (2009). Genetic behavior for some root characters and their relation to some other characters under drought condition in rice (Oryza sativa L.). J. Agric. Sci. Mansoura. Univ., 34(2): 1153-1172.

Abdel-Moneam, M.A. ; M.S. Sultan ; S.A. Hammoud ; A.G. Hefena ; C. Barutçular and A. El-Sabagh (2016). Studies on heterosis for qualitative and quantitative traits in rice. J. Of Animal \&Plant Sci., 30(2): 4736-4747.

Butany, W.T. (1961 ) . Mass emasculation in rice. Inster. Rice Comm. Newsletter 9: 9-13.

El-Gamal, W.H. (2013). Inheritance of some traits related to drought tolerance in rice. Ph.D. Thesis, Fac. of Agric. Mansoura Univ., Egypt, pp. 130.

Falconer, D.S. and F.C. Mackey ( 1996) . Introduction to quantitative genetics. Fourth Edition. Longman. New York.

FAO (2018). Food and Agriculture Organization. Faostat, FAO Statistics Division, March, 2018.

Gaballah, M.M. (2009). Studies on physiological and morphological traits associated with drought resistance in rice (Oryza sativa L.). Ph.D. Thesis, Agron., Dept., Fac. Agric., KafrelSheikh Univ., Egypt, pp. 212.

Ghosh, S.C. ; P.K. Chandrakar ; N.K. Rastogi ; S. Deepak and A.K. Sarawgi (2014). Combining ability analysis using CMS breeding system for developing hybrids in rice (Oriza sativa $L_{\text {. }}$ ). Bangladesh J. Agric. Res., 37(4): 583-592.

Griffing, B. (1956). A generalized treatment of the use of diallel crosses in quantitative inheritance. Heredity, 10: 31-50.

Hassan, H.M.; E.F.A. Arafat and A. EL Sabagh (2016). Genetic studies on vegetative and agronomic traits in rice (Oryza sativa L.) under water stress conditions. J. Agri. Biotech., 1(02): 76-84.

Hefena, A.G. ; M.S. Sultan ; M.A Abdel-Moneam ; S.A. Hammoud ; C. Barutçular and A. EL-Sabagh (2016). Genetic variability, heritability and genetic advance for yield and associated traits in $\mathrm{F}_{2}$ rice population. J. of Agric. Biotech., 1(2): 49-58.

Jodon, N.E. (1938) . Experiment on artificial hybridization of rice. J. Amer. Soci. Argon., 30: 249-305.

Karpagam, V.; S. Jebaraj and S. Rajeswari (2016). Combining ability and heterosis analysis for drought tolerant traits in rice (Oryza sativa L.). J. Appl. \& Nat. Sci., 8 (2): 674 - 682.
Malemba, G.M. ; F.M. Nzuve ; J.M. Kimani ; M.F. Olubayo and J.W. Muthomi (2017). Combining ability for drought tolerance in upland rice varieties at reproductive stage. J. of Agric. Sci., 9(3): 138150 .

Manickavelu, A. ; N. Nadarajan; S.K. Ganesh and R.P. Gnanamalar (2006). Genetic analysis of biparental progenies in rice (Oryza sativa L.). Asian J. of Plant Sci., 5(1): 33-36.

Pandey, S. ; H. Bhandari ; R. Sharan ; S. Ding ; P. Prapertchob ; D. Naik and K.S. Taunk (2005). Coping with drought in agriculture of developing counties: insights from rice farming in Asia. In: Proc. of the $2^{\text {nd }}$ Intern. Conf. on Integrated Approaches to Sustain and Improve Plant Production under Drought Stress. Univ. of Rome, "La Sapienza", Rome, Italy, Sept, 24-28.

Ramesh, C. ; C.D. Raju ; C.S. Raju and N.R. Varma (2018). Combining ability and gene action in hybrid rice. Intern. J. Pure App. BioSci., 6(1): 497510 .

Sedeek, S.E.M. (2006). Breeding studies on rice. Ph.D. Thesis, Faculty of Agriculture, Kafr El-Sheikh, Tanta University, Egypt.

Singh, R.K. and B.D. Chaudhary. ( 1979) . Biometrical Methods in Quantitative Genetic Analysis. Kalyani Publ., New Delhi.

Sultan, M.S. ; M.A. Abdel-Moneam; A.B. El-Abd and S.A. El-Naem (2014b). Inheritance of some root and grain quality traits in rice under water deficiency conditions. J. of Agron., 13 (3): 89-99.

Sultan, M.S. ; M.A. Andel-Moneam ; A.B. El-Abd and S.A. El-Naem (2014a). Inheritance of grain yield and its related traits in rice under water deficiency conditions. J. Plant Prod. Mansoura Univ., 5(1):4152.

Sultan, M.S. ; M.I. Abo Youssef ; M.A. Abdel-Moneam ; M.M. El-Shenawy (2014c). Combining ability studies for grain yield and some associated characters in some rice lines. J. Plant Prod. Mansoura Univ., 5 (4): 685-697.

Tiwari, D.K.; P. Pandey; S.P. Giri and J.L. Dwivedi (2011). Heterosis studies for yield and its components in rice hybrids using CMS system. Asian J. Plant Sci., 10(1): 29-42.

Wynne, J.C., D.A. Emery and P. W. Rice. (1970). Combining ability estimates in Arachis hypogea L. II- Field performance of $F_{1}$ hybrids. Crop Sci., 10.

Yadav, P.; N.R. Rangare; P.J. Anurag and A.K. Chaurasia (2010). Quantitative analysis of rice (Oryza sativa L.) in Allahabad agro climate zone. J. Rice Res., 3(1): 16-18.

Yogameenashi, P.; N. Nadarajan and A. Sheeba (2003). Evaluation of varieties and land races for drought tolerance in rice (Oryza sativa L.). Indian J. Genet., 63(4): 299-303.

Zhu, F., ; Y. Cai ; J. Bao and H. Corke (2010). Effect of irradiation on phenolic compounds in rice grain. Food Chem., 120: 74-77. 
القدرة على التآلف وقوة الهجين لبعض صفات الجذر والصفات الخضرية في الأرز تحت ظروف الري الطبيعي والاجهاد

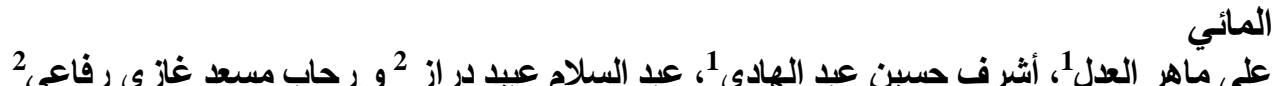

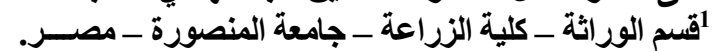

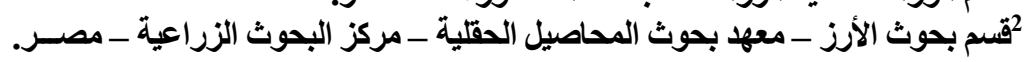

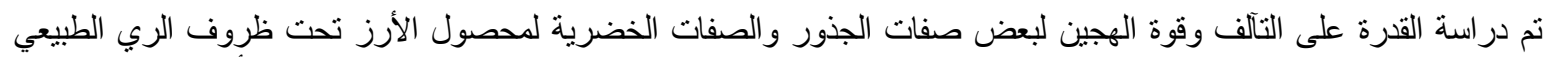

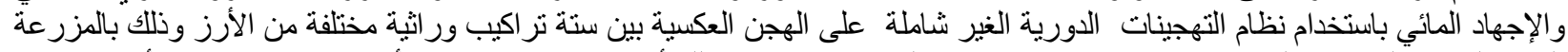

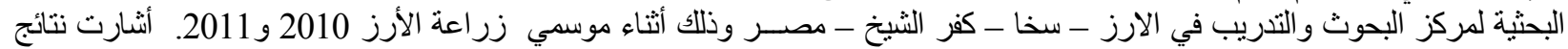

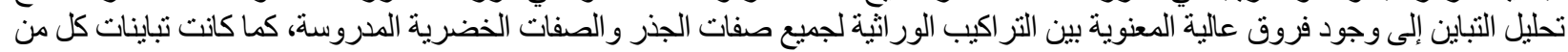

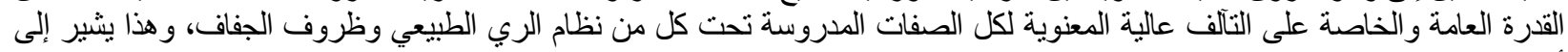

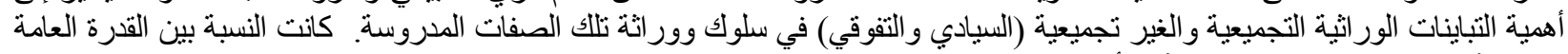

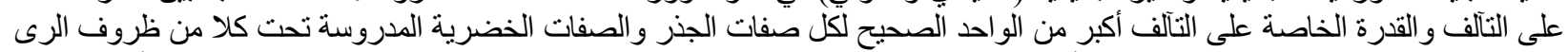

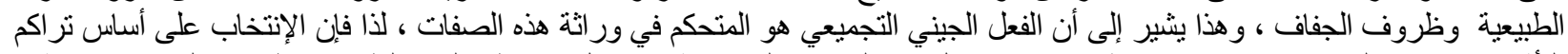

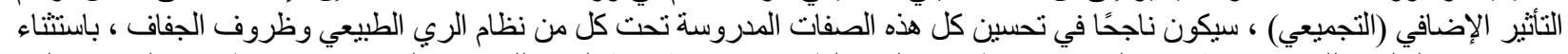

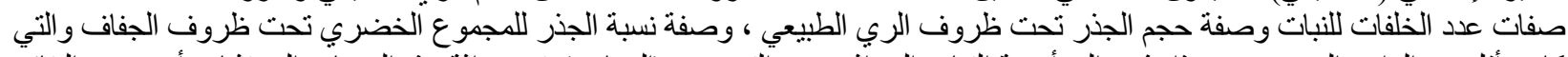

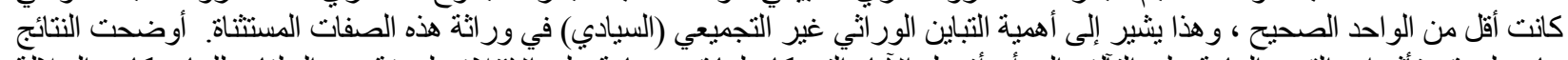

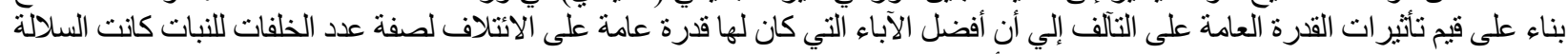

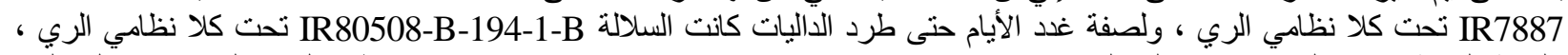

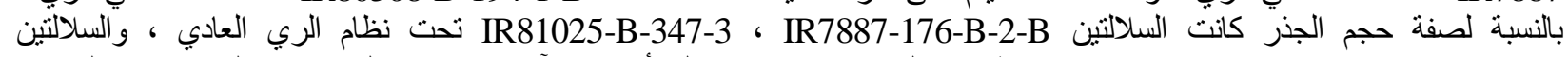
IR7887-176-B-2-B ، AZUCENA

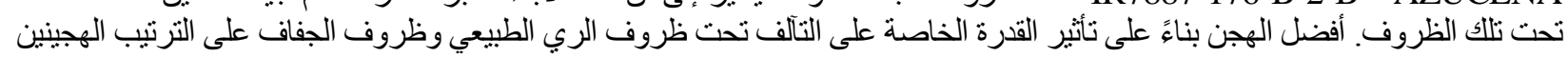

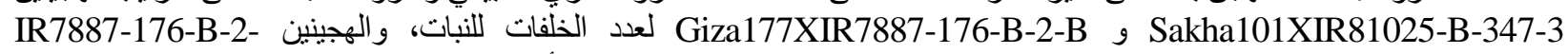

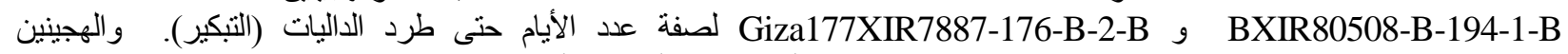
Sakha101X IR80508-B-194-1-B لصفة حجم الجذر، و الهجينين Sakha101XIR7887-176-B-2-B و ولجين Sakha101XAzucena

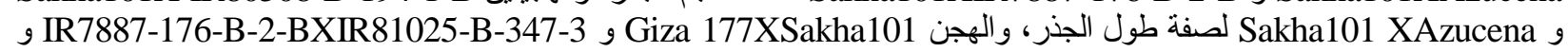
Giza177XAZUCENA لصفة ارتفاع النبات (القصر) تحت كلا نظامي الري ، والهجن IR80508-B-194-BXIR81025-B-k347-3 IR 7887- ، Sakha101 X IR80508-B-194-1-B ، Sakha101 X IR7887-176-B-2-B ، Sakha101XAZUCENA ،

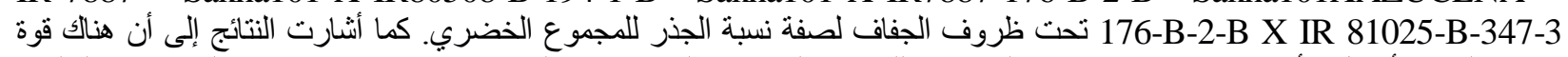

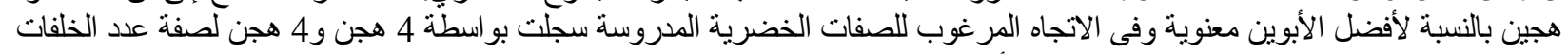

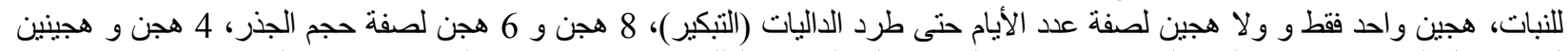

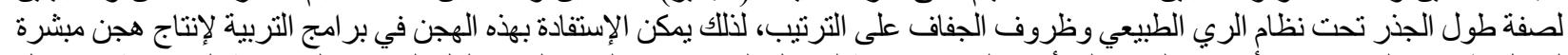

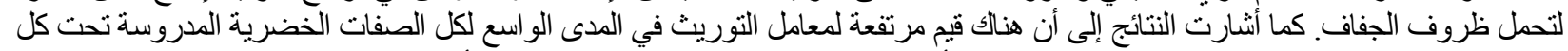
من نظام الري الطبيعي وظروف الجفاف، وهذا يثير إلى أن التباينات الور اثثية تمثل الجز ء الرئيسي والأكبر في التباينات المظهرية (الكلية). 